\title{
Erratum to: Relating the CMSSM and SUGRA models with GUT scale and super-GUT scale supersymmetry breaking
}

\author{
Emilian Dudas $^{1,2,3}$, Yann Mambrini ${ }^{3}$, Azar Mustafayever,a ${ }^{4}$, Keith A. Olive ${ }^{4}$ \\ ${ }^{1}$ Department of Physics, Theory Division, 1211, Geneva 23, Switzerland \\ ${ }^{2} \mathrm{CPhT}$, Ecole Polytechnique, 91128 Palaiseau, France \\ ${ }^{3}$ Laboratoire de Physique Théorique, Université Paris-Sud, 91405 Orsay, France \\ ${ }^{4}$ William I. Fine Theoretical Physics Institute, School of Physics and Astronomy, University of Minnesota, Minneapolis, MN 55455, USA
}

Published online: 15 May 2013

(C) Springer-Verlag Berlin Heidelberg and Società Italiana di Fisica 2013

Erratum to: Eur. Phys. J. C (2012) 72:2138

DOI 10.1140/epjc/s10052-012-2138-3

In the original version of the paper, there was an ambiguity between the value of $\mu$ before and after the shift due to the Giudice-Masiero (GM) term. Here, we will clarify the equations which were affected. We define $\mu_{0}$ as the $\mu$-term in the superpotential defined at the input universality scale $M_{\text {in }}$. $\mu\left(M_{\text {in }}\right)$ will refer to the $\mu$-term after the shift induced by the GM contribution to the Kähler potential also defined at the input scale. Then Eq. (12) becomes

$\mu\left(M_{\text {in }}\right)=\mu_{0}+c_{H} m_{0}$.

Similarly, $\mu B\left(M_{\mathrm{in}}\right)$ is defined as

$\mu B\left(M_{\text {in }}\right)=\mu_{0} B_{0}+2 c_{H} m_{0}^{2}$,

which replaces Eq. (13). As a consequence, we would find

$B\left(M_{\text {in }}\right)=\left(A_{0}-m_{0}\right) \mu_{0} / \mu\left(M_{\text {in }}\right)+2 c_{H} m_{0}^{2} / \mu\left(M_{\text {in }}\right)$.

This clarification affects the result only in Sect. 2 of the paper. For $M_{\mathrm{in}}=M_{\mathrm{GUT}}$, and when the Giudice-Masiero term (11) is included [15], one can deduce the (GUT) boundary conditions for $\mu$ and $B$ :

$$
\begin{aligned}
\mu\left(M_{\mathrm{GUT}}\right)= & \mu_{0}+c_{H} m_{0}, \\
B\left(M_{\mathrm{GUT}}\right)= & \left(A_{0}-m_{0}\right) \mu_{0} / \mu\left(M_{\mathrm{GUT}}\right) \\
& +2 c_{H} m_{0}^{2} / \mu\left(M_{\mathrm{GUT}}\right) .
\end{aligned}
$$

This allows us to solve for $c_{H}$ where we obtain an equation similar to Eq. (30):

$c_{H}=\left(B\left(M_{\mathrm{GUT}}\right)-A_{0}+m_{0}\right) \mu\left(M_{\mathrm{GUT}}\right) /\left(3 m_{0}^{2}-A_{0} m_{0}\right)$.

These changes affect the contours in Figs. 2-4. In Fig. 2, with $A_{0}=0$, all contour labels should be multiplied by 2/3. In Fig. 3, with $A_{0}=2.5 m_{0}$, all contours should be multiplied by 4.0. In Fig. 4a, with $A_{0}=0$, all contour labels should be multiplied by $2 / 3$. Finally, in Fig. $4 b$, with $A_{0}=2.0 m_{0}$, all contour labels should be multiplied by 2.0 .

All results and figures in Sects. 3 and 4 remain unaffected.

The online version of the original article can be found under doi:10.1140/epjc/s10052-012-2138-3.

a e-mail: azar@phys.hawaii.edu 